\title{
Structural phase transition in carbon nanotube bundles under pressure
}

\author{
M. J. Peters \\ Department of Physics and Astronomy, Appalachian State University, Boone, North Carolina 28608 \\ L. E. McNeil, Jian Ping Lu, and Daniel Kahn \\ Department of Physics and Astronomy, University of North Carolina at Chapel Hill, Chapel Hill, North Carolina 27599
}

(Received 22 July 1999)

\begin{abstract}
The Raman shifts as functions of pressure were measured for the radial breathing mode and tangential modes in carbon nanoropes containing $(10,10)$ and $(17,0)$ single-walled tubes. An abrupt decrease in the rate of change of the Raman shift with pressure for the tangential modes, and the disappearance of the radial breathing mode, were observed at approximately $1.7 \mathrm{GPa}$. These changes in the Raman spectrum result from a structural phase transition as the nanoropes are compressed. Theoretical calculations using the empirical force method confirm this transition, as indicated by a calculated discontinuity in the lattice constants and unit cell volume at $1.7 \mathrm{GPa}$.
\end{abstract}

\section{INTRODUCTION}

While single-walled carbon nanotubes (SWNTs) were discovered relatively recently, ${ }^{1}$ their electronic ${ }^{2-5}$ and mechanical $^{6-10}$ properties have made them targets of extensive study. Vibrational spectroscopy, and Raman spectroscopy in particular, has proved to be a useful tool in examining these structures, ${ }^{9,11-20}$ and theoretical work ${ }^{2,7,8,21-27}$ has produced models to explore the vibrations of the tubes for various structures as well as the interactions between the tubes. As an experimental probe of the tube interactions, Raman spectroscopy at high pressure is a particularly fruitful technique. ${ }^{12}$ In this paper, we present a high-pressure Raman study of single-walled carbon nanotube bundles which indicates that the morphology of the tube bundles undergoes a structural phase transition at approximately $1.7 \mathrm{GPa}$. We also present theoretical calculations confirming the existence of this transition, and the magnitude of the transition pressure.

\section{EXPERIMENTAL DETAILS}

The SWNT sample was created by laser ablation using a $\mathrm{Nd}$ :YAG pulsed laser operating at $110 \mathrm{~mJ} /$ pulse at a wavelength of $10640 \AA$. The furnace temperature was $1150{ }^{\circ} \mathrm{C}$ and the argon flow rate was 140 SCCM. The target was composed of $99.4 \%$ carbon, $0.3 \%$ cobalt, and $0.3 \%$ nickel. Using SEM and TEM, the estimated purity of the resulting SWNT sample was found to be $60-70 \%$. The remaining amount of sample was comprised of the $\mathrm{Co} / \mathrm{Ni}$ catalysts as well as carbon in the forms of nanoparticles, amorphous carbon, and graphite. Details of the fabrication have been published elsewhere. ${ }^{28}$

The pressure measurements were made using a MerrillBassett diamond-anvil cell. The sample chamber was created by a hole approximately $0.25 \mathrm{~mm}$ in diameter, cut in a 0.1 mm thick gasket made of Inconel. This chamber was centered between two $\frac{1}{4}$ carat diamonds. The pressure exerted was hydrostatic, achieved with the use of a pressure transmitting medium of 4:1 methanol to ethanol. Measurement of pressure in the cell was made using ruby fluorescence. ${ }^{29}$
The Raman spectra were measured using a micro-Raman illuminator attached to a Dilor $X Y$ triple spectrometer. The laser source used was a Spectra Physics 2017 argon ion laser set to the $5145 \AA$ line with an average power of $60 \mathrm{~mW}$ on the upper diamond. The microscope focused the incident beam to a spot size of $50 \mu \mathrm{m}$ and the backscattered light was collected $180^{\circ}$ from the direction of incidence. The resolution of the spectrometer is $0.5 \mathrm{~cm}^{-1}$. A CCD camera cooled with liquid nitrogen collected the data, which was then analyzed using a least-squares fit to a sum of Lorentzians.

\section{RESULTS}

\section{A. Identification of modes}

Though many more Raman modes are present in the full spectrum of SWNTs, this research focused on modes in two primary ranges: a low energy range between $150-200 \mathrm{~cm}^{-1}$ and a high energy range between $1500-1650 \mathrm{~cm}^{-1}$. The lower energy range contains the radial breathing mode; the higher energy range contains the modes associated with the graphitelike characteristics of the SWNTs. In the next two sections of this paper we use Raman measurements of the radial breathing mode and $\mathrm{x}$-ray diffraction of the tube bundles to identify tubes in our sample. We then use these tubes to assign the modes measured in the higher energy range, comparing calculated to measured frequencies. In both cases, we include a discussion of the resonance effects in SWNTs. ${ }^{22}$

\section{Breathing mode}

The breathing mode of a SWNT is a purely radial vibration with all atoms moving perpendicular to the length of the tube as seen in inset of Fig. 1. A typical Raman spectrum of the breathing mode of our SWNT sample is shown. Only one mode centered at $181 \pm 3 \mathrm{~cm}^{-1}$ is measured. As has been reported, ${ }^{22,30}$ the frequency of the breathing mode is sensitive to the diameter of the tube. For example, the difference in the breathing mode frequencies of the $(10,10)$ and $(11,11)$ tubes 


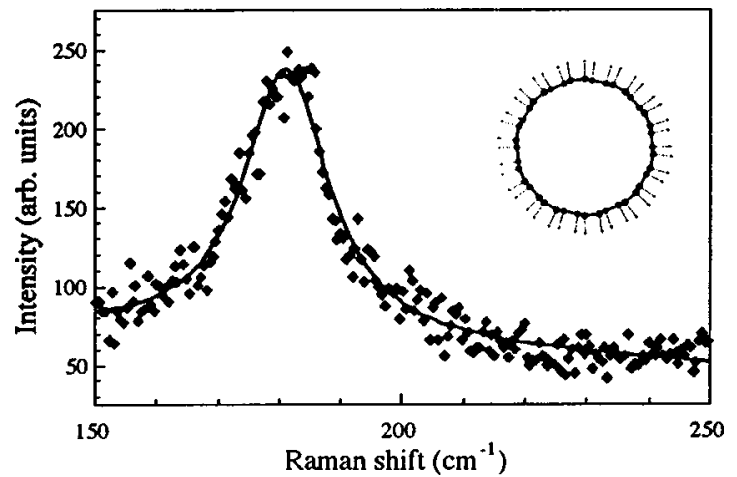

FIG. 1. Raman spectrum of radial breathing mode fit with a Lorentzian function. Inset: Molecular dynamic simulation of carbon atoms in $(10,10)$ tube undergoing radial breathing mode vibration.

with diameters of 13.4 and $14.9 \AA$, respectively, is $17 \mathrm{~cm}^{-1}$ while there is no measurable difference between the breathing mode frequencies of the $(10,10)$ and $(17,0)$ tubes with diameters of 13.4 and $13.2 \AA$, respectively. ${ }^{30}$ Thus, while multiple breathing modes found in a Raman spectrum indicate that tubes of differing diameters exist in the sample, the presence of a single mode does not necessarily indicate that the sample consists of one type of tube.

Resonance effects seen in SWNTs (Ref. 22) further complicate the interpretation of the Raman spectrum. The intensities of modes are dependent upon the excitation wavelength. This effect is discussed extensively by Rao et al. ${ }^{20}$ who show that when the excitation wavelength is changed, the intensities can change so dramatically that prominent modes may disappear and modes that were absent may appear. This effect is seen throughout the spectrum. Therefore, even though a sample contains tubes of differing diameters, modes arising from some of the tubes may not be observed.

Our spectra were taken with an excitation wavelength of $5145 \AA$. Focusing on the low energy range and the radial breathing mode, we measured one mode at $181 \pm 3 \mathrm{~cm}^{-1}$. This is in good agreement with other experimental work ${ }^{20}$ in which only one mode was also measured using the same excitation. Implications of the resonance effect on the modes found in the high energy region will be discussed in the following section.

$\mathrm{X}$-ray analysis on the sample revealed that the average diameter of the tubes in the sample is $13.5 \AA$. The $\mathrm{x}$-ray spectrum was modeled with $(10,10)$ tubes of differing bundle sizes. The width of the $\mathrm{x}$-ray diffraction peak is greater than that of the simulated spectrum with just $(10,10)$ tubes, indicating that more than one kind of tube exists in the sample. Using the Scherrer formula, the coherence length was found to be $80 \AA$. This indicates that the bundle sizes in the sample range from 80 to $200 \AA$. Because of the circular electron distribution in the tube cross section, $\mathrm{x}$-ray analysis cannot distinguish chirality, but sorts only by tube diameter. Using the expression relating values of $n$ and $m$ to tube diameter, ${ }^{31}$ an average tube diameter of $13.5 \AA$ was found to match $(10,10)$ armchair tubes and $(17,0)$ zigzag tubes.

Calculations of the radial breathing mode in SWNTs were made by Kurti et al. ${ }^{30}$ using local density approximation theory. Breathing modes of both armchair $(n, n)$ and zigzag $(n, 0)$ tubes were calculated for a variety of values of $n$. These calculations were made considering isolated tubes that were

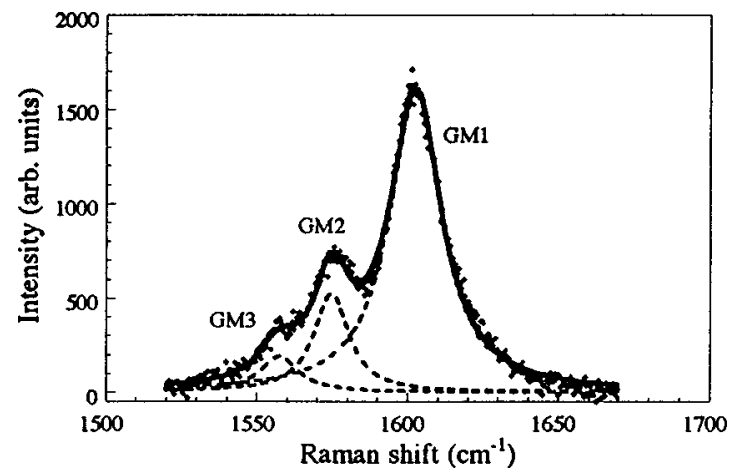

FIG. 2. Raman spectrum of graphitelike modes indicating the three individual modes labeled: GM1, GM2, GM3.

infinitely long. The frequencies for the breathing mode calculated for the $(10,10)$ and $(17,0)$ tubes are similar: $175 \mathrm{~cm}^{-1}$ for the $(10,10)$ tube, $178 \mathrm{~cm}^{-1}$ for the $(17,0)$ tube. Calculations of the difference in mode frequency in isolated tubes vs bundles ${ }^{21}$ show that the frequency of the radial breathing mode in a $(10,10)$ tube increases by approximately $6 \mathrm{~cm}^{-1}$ when the tubes present are in bundles. Assuming the difference would be the same for the $(17,0)$ zigzag tube, this would place the frequencies calculated using the LDA theory at 181 and $183 \mathrm{~cm}^{-1}$ for the $(10,10)$ and $(17,0)$ tubes, respectively. Both of these frequencies are consistent with the breathing mode frequency we measured, $181 \pm 3 \mathrm{~cm}^{-1}$. Again, it is noted that the resonance effects are also involved in any attempt to identify the tubes present in the sample. ${ }^{20}$ However, using the information given above, other experimental data taken at the same excitation wavelength, and $\mathrm{x}$-ray diffraction analysis, we conclude in this paper that we have at minimum $(10,10)$ armchair tubes and $(17,0)$ zigzag tubes.

\section{Graphitelike modes}

We now move to identify the modes in the higher energy range. Raman measurements show modes between 1500 and $1650 \mathrm{~cm}^{-1}$. The highest energy mode in graphite occurs in this range, at $1582 \mathrm{~cm}^{-1} \cdot{ }^{31}$ Because the tubes are essentially graphene sheets rolled into cylinders, the modes in this higher-energy region are referred to as graphitelike modes. Three peaks (labeled GM1, GM2, and GM3) were measured in our spectra as can be seen in Fig. 2. These are consistent with those seen by Rao et al. ${ }^{20}$ and Venkateswaran et al. ${ }^{12}$

Calculations of Raman and IR active modes for many isolated tubes with a range of $n$ and $m$ values were made by Kahn and $\mathrm{Lu}^{21}$ using tight-binding methods. The actual values for frequencies using tight-binding methods are difficult to determine because the method overestimates the energies by approximately $6 \%$. However, the spacings between the modes and the assignments of symmetry to frequency are well determined. To relate our measured data to the calculated values of frequencies, we scaled the calculated data, equating the highest frequencies in the measured and calculated data. Thus the highest calculated frequency was scaled to $159 \mathrm{~cm}^{-1}$, and the same scaling factor was then applied to the other calculated frequencies. Table I presents the scaled calculated frequencies for both the $(10,10)$ and $(17,0)$ tubes and the measured frequencies at $0.25 \mathrm{GPa}$, which are in good 
TABLE I. Measured and calculated Raman frequencies of graphitelike modes. Calculated values were scaled so the highest calculated frequency is equal to the highest measured frequency. Symmetry assignments and $(n, m)$ values of tubes were determined by calculations using tight-binding methods.

\begin{tabular}{ccc}
\hline \hline Peak labels & $\begin{array}{c}\text { Measured frequencies } \\
\left(\mathrm{cm}^{-1}\right)\end{array}$ & $\begin{array}{c}\text { Scaled calculated } \\
\text { frequencies }\left(\mathrm{cm}^{-1}\right), \text { mode } \\
\text { assignments, and tubes }\end{array}$ \\
\hline GM1 & 1591 & $1591 E_{2 g}(10,10)$ \\
& & $1577 E_{1 g}(17,0)$ \\
GM2 & 1563 & $1571 A_{1 g}(10,10)$ \\
& & $1566 E_{1 g}(10,10)$ \\
GM3 & 1550 & $1565 A_{1 g}(17,0)$ \\
& & $1551 E_{2 g}(17,0)$ \\
\hline \hline
\end{tabular}

agreement with other experimental results reported. ${ }^{12}$ Data taken at $0.25 \mathrm{GPa}$ were used because the GM3 peak could not be resolved at atmospheric pressure.

Once the highest measured mode (GM1) was identified as the $E_{2 g}$ mode of the $(10,10)$ tubes and the rest of the calculated frequencies were scaled accordingly, the lowest measured mode (GM3) could be identified as the $E_{2 g}$ mode of the $(17,0)$ tubes. The agreement of the scaled calculated frequency with the measured frequency for this mode is excellent. The GM2 peak is a weighted combination of the $A_{1 g}$ and $E_{1 g}$ modes of the two tubes. As discussed earlier in this section, the relative intensities of the modes is dependent on a resonance effect. This, in conjunction with the relative abundance of the two kinds of tubes and polarization effects, ${ }^{22}$ will affect the weighting of the modes involved in peak GM2. These symmetry identifications of the measured frequencies are consistent with shifts in the frequencies observed as pressure is applied to the tubes. These results are presented in the next section.

\section{B. Pressure results}

Raman measurements at high pressure were made in the range from atmospheric to $5.3 \mathrm{GPa}$. While similar experiments have been performed, ${ }^{12}$ our research has revealed a structural phase transition at approximately $1.7 \mathrm{GPa}$ that has

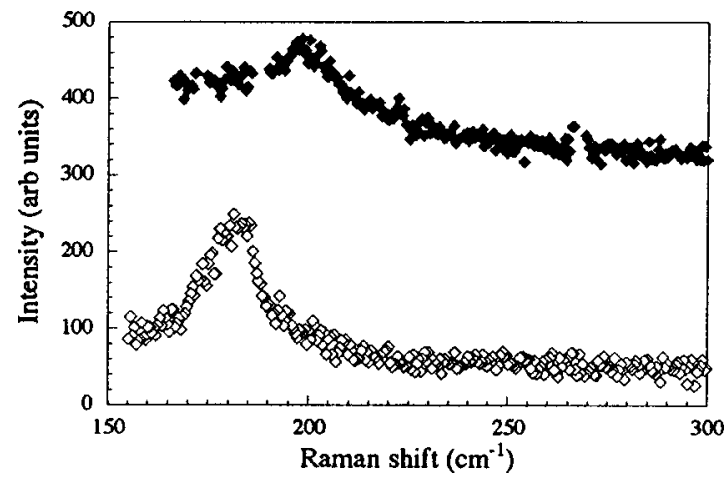

FIG. 3. Spectra of radial breathing mode at atmospheric pressure (open diamonds) and $1.5 \mathrm{GPa}$ (filled diamonds) showing move to higher Raman shift as well as a reduction in intensity with increasing pressure.

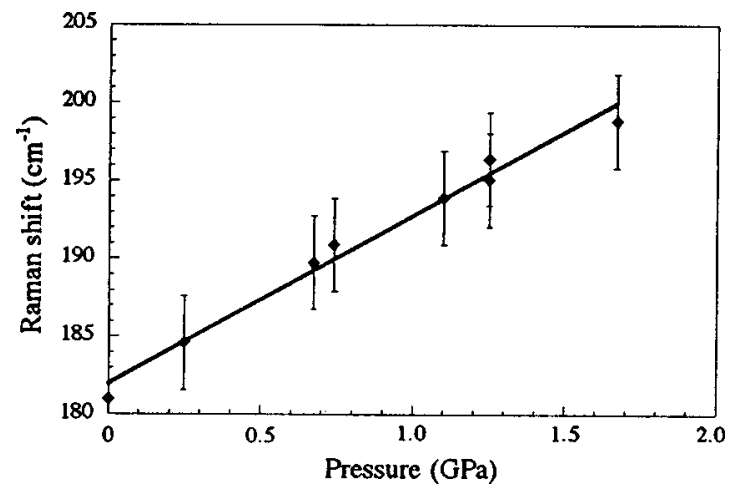

FIG. 4. Raman shift in radial breathing mode as a function of pressure. The slope is $10.1 \pm 1.2 \mathrm{~cm}^{-1} / \mathrm{GPa}$.

not been previously observed. The effect of the pressure on the breathing mode and graphitelike modes are discussed separately.

\section{Breathing mode}

Figure 3 shows the Raman shift of the radial breathing mode at atmospheric pressure and $1.5 \mathrm{GPa}$. Rayleigh scattered light from the diamonds, coupled with a small sample volume, make signal detection of the low-frequency modes difficult at high pressures. To eliminate spurious signals due to ambient nitrogen, measurements in this frequency region were taken with the cell in a He atmosphere.

We measured the shift of the frequency as a function of pressure to be $10.1 \pm 1.2 \mathrm{~cm}^{-1} / \mathrm{GPa}$; see Fig. 4. This shift was calculated for low pressures (less than $0.5 \mathrm{GPa}$ ) by Kahn and Lu. ${ }^{21}$ These calculations show a rate of change in frequency with applied pressure of $12.0 \mathrm{~cm}^{-1} / \mathrm{GPa}$, in good agreement with the experimental results.

Raman measurements of the radial breathing mode for pressures above approximately $1.7 \mathrm{GPa}$ were difficult to make. This difficulty was due to deteriorating signal from this mode as a result of a structural change that will be explained later in this paper.

\section{Graphitelike modes}

As with the radial breathing mode, the graphitelike modes also shift to higher frequency with increasing pressure. See Fig. 5. The data for the higher-energy graphitelike modes are

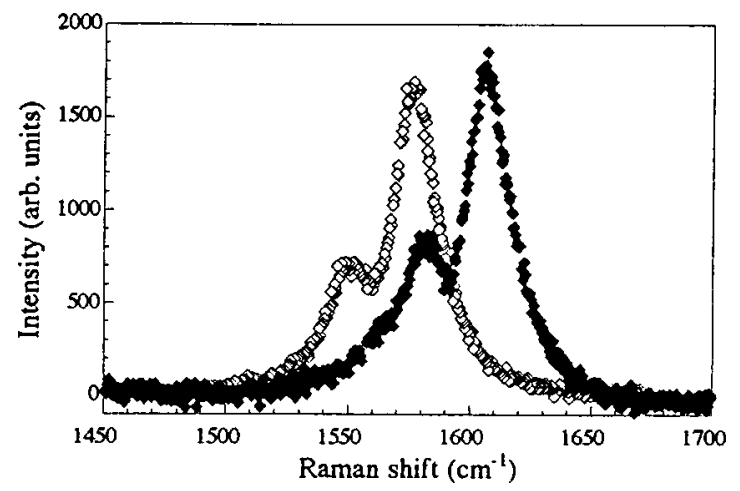

FIG. 5. Spectra of graphitelike modes at atmospheric pressure (open diamonds) and 1.5 GPa (filled diamonds) showing shift to higher energy with increasing pressure. 


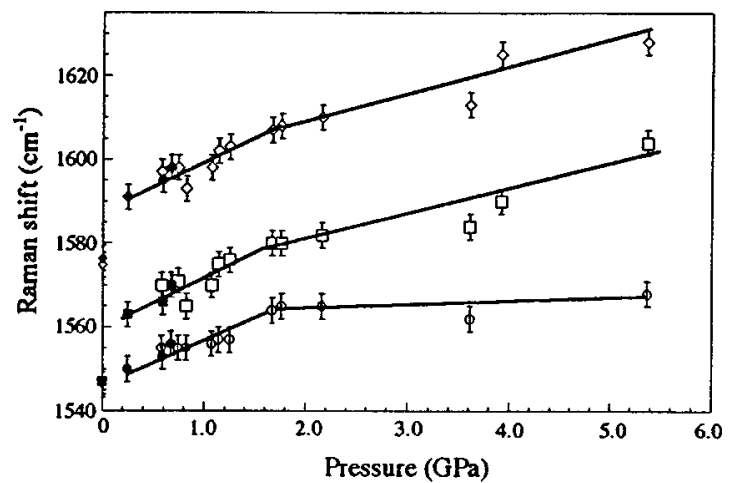

FIG. 6. Raman shift vs pressure for graphitelike modes: GM1 (diamond), GM2 (square), and GM3 (circle). Open symbols indicate data points taken as pressure is increased. Closed symbols indicate data points taken as pressure is reduced.

divided into two regions, low pressure and high pressure, with the boundary between the two regions falling approximately at $1.7 \mathrm{GPa}$. The response to pressure of these modes is very different in these two regions. See Fig. 6 for the full range of pressure measurements.

In the low-pressure region, the change in peak position as a function of pressure is found to be similar for all three peaks. The slopes are, from highest to lowest frequency modes, $10.1 \pm 1.1 \mathrm{~cm}^{-1} / \mathrm{GPa}$ (GM1), $11.0 \pm 1.2 \mathrm{~cm}^{-1} / \mathrm{GPa}$ (GM2), and $6.1 \pm 0.1 \mathrm{~cm}^{-1} / \mathrm{GPa}$ (GM3). The results of calculations by $\mathrm{Kahn}$ and $\mathrm{Lu}^{21}$ in which the authors present peak positions vs pressure for the three modes in the $(10,10)$ armchair tubes are in good agreement with the experimental data: $11.0 \mathrm{~cm}^{-1} / \mathrm{GPa}, 12.1 \mathrm{~cm}^{-1} / \mathrm{GPa}$, and $9.0 \mathrm{~cm}^{-1} / \mathrm{GPa}$. It should be noted that all the slopes are larger than that found in the high-energy mode of bulk graphite: $5.00 \mathrm{~cm}^{-1} / \mathrm{GPa}^{32}$

These graphitelike modes display a very different response to pressure in the region we labeled high pressure, above $1.7 \mathrm{GPa}$. The rate of change of frequency with pressure decreased for all three peaks. The two higher energy peaks show a rate of change in position with pressure of $5.8 \pm 0.2 \mathrm{~cm}^{-1} / \mathrm{GPa}$ (GM1) and $5.9 \pm 0.1 \mathrm{~cm}^{-1} / \mathrm{GPa}$ (GM2) These slopes compare well with that found in bulk graphite, $5.00 \mathrm{~cm}^{-1} / \mathrm{GPa}^{32}$ The lower-energy peak shows a very small change in frequency with pressure $0.7 \pm 0.1 \mathrm{~cm}^{-1} / \mathrm{GPa}$ (GM3).

These high pressure effects are reversible, but only slowly. If data are taken immediately after the pressure in the cell is released, the frequencies recorded fall between the atmospheric-pressure value the value measured at the last applied pressure. After approximately $30 \mathrm{~min}$, when measurements are taken again, the frequencies have returned (within error bars) to the atmospheric values measured outside the cell prior to any pressure application. This time dependence has not yet been explored quantitatively.

\section{Pressure results in terms of spacing between tubes}

The response to pressure of the tubes was also analyzed as a function of spacing between the tubes using the bulk modulus calculated by Lu. ${ }^{10}$ Figure 7 shows the Raman shift versus spacing data for the breathing mode and graphitelike modes. The rates at which the frequency changes with decreasing spacing are given in Table II.

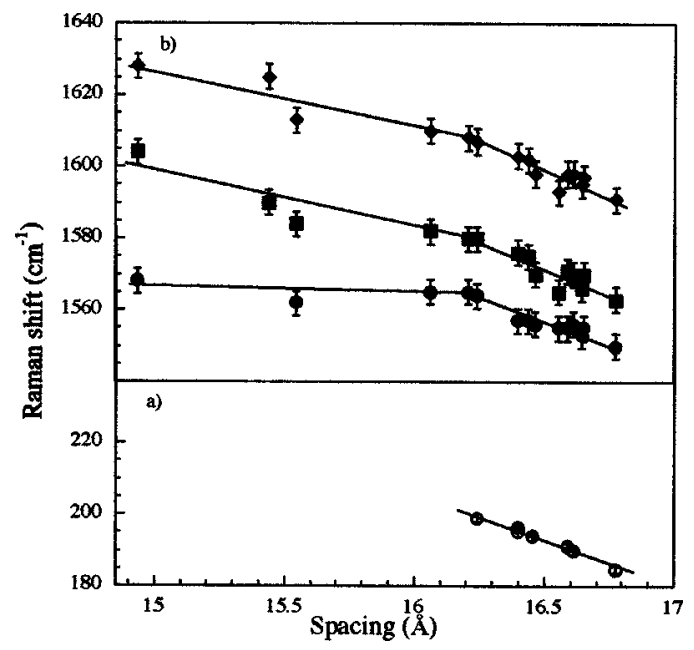

FIG. 7. Raman shift vs spacing between tubes for (a) the radial breathing mode and (b) the graphitelike modes (GM1=diamond; $\mathrm{GM} 2=$ square; GM3=circle). Smaller spacing indicates higher pressure.

\section{DISCUSSION AND CONCLUSIONS}

This study of single-wall nanotubes under pressure has examined the tube-tube interactions in bundles containing $(10,10)$ armchair and $(17,0)$ zigzag tubes, as manifested in the Raman-active vibrational modes. A discontinuity was measured in the Raman shift vs pressure for the higherfrequency graphitelike modes. In the low-pressure region, all the measured modes respond to pressure at the same rate of change, approximately $10 \pm 1 \mathrm{~cm}^{-1} / \mathrm{GPa}$. As noted earlier, this is approximately twice that found in bulk graphite. Because the intensity of the radial breathing mode decreases substantially as the tubes are forced closer together, it was not observed in the high-pressure region. However, the positions of the three other Raman peaks, in the higher-energy range, do not continue to change at this same rate at the highest pressures. Instead, two of the peaks, GM1 and GM2, show a change in frequency with pressure comparable to that measured in bulk graphite, while GM3 exhibits very little change in Raman shift with increasing pressure.

The pressure dependence of these modes is consistent with the identification of the symmetries corresponding to the peaks. The highest-frequency mode for each type of tube is the one for which the atoms move circumferentially. For the armchair tube $(10,10)$, this mode has $E_{2 g}$ symmetry; for the zigzag tube $(17,0)$, this mode has $E_{1 g}$ symmetry. The lowest-frequency mode in each case is the one in which the atoms move along the longitudinal axis of the tube. Because

TABLE II. Rates of change of Raman shift as a function of spacing between tubes. The change in the Raman shift is much greater in the high pressure region for all three modes.

\begin{tabular}{ccc}
\hline \hline Mode & $\begin{array}{c}\text { Slope } \\
\text { in low pressure region } \\
\left(\mathrm{cm}^{-1} / \AA\right)\end{array}$ & $\begin{array}{c}\text { Slope } \\
\text { in high pressure region } \\
\left(\mathrm{cm}^{-1} / \AA\right)\end{array}$ \\
\hline GM1 & $16.3 \pm 0.2$ & $26.7 \pm 0.2$ \\
GM2 & $16.6 \pm 0.2$ & $29.1 \pm 0.2$ \\
GM3 & $1.8 \pm 0.2$ & $16.1 \pm 0.2$ \\
\hline \hline
\end{tabular}




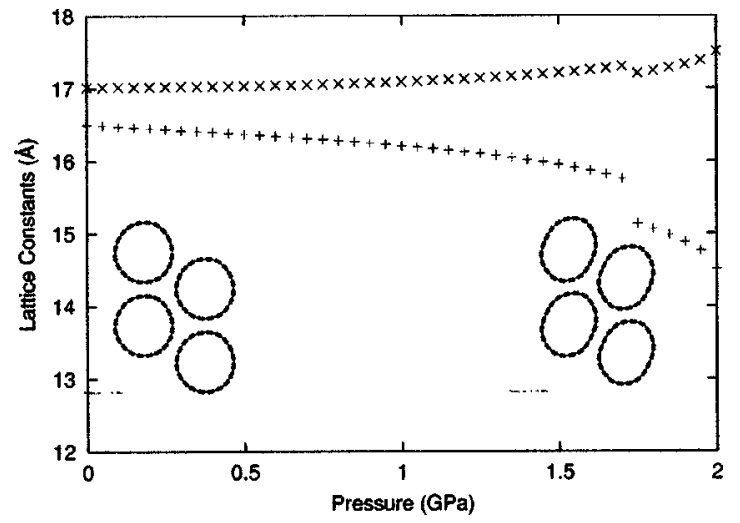

FIG. 8. Plane lattice constants vs pressure for $(10,10)$ nanorope. Insets: Cross section of tubes at atmospheric pressure and 1.85 GPa.

the structures of the two tubes are symmetry-related by a $90^{\circ}$ rotation, this longitudinal motion is present in the $E_{1 g}$ for armchair tubes and the $E_{2 g}$ modes for zigzag tubes. The circumferential modes have components of motion perpendicular to the tube axis; therefore, these modes are most sensitive to the spacing between the tubes. The GM1 peak is identified as the circumferential $E_{2 g}$ mode of the armchair $(10,10)$ tube. The GM2 peak is identified as a mixture of circumferential and radial $(A 1 \mathrm{~g})$ modes, all containing components in the direction perpendicular to the tube axis. The GM3 peak is identified as the $E_{2 g}$ mode of the zigzag $(17,0)$ tube and, therefore, represents motion along the direction of the tube. The difference in the response to pressure of the GM3 peak with respect to the other two occurs because the GM3 peak results from vibrational motion that is almost entirely longitudinal. Therefore, this mode is less sensitive to the proximity of other tubes. Once the transitional pressure is reached, the GM3 shows very small change in energy with applied pressure, much lower than that found in bulk graphite. This may be related to the fact that the tubes, unlike the planes in graphite, are not in lateral registry.

\section{STRUCTURAL PHASE TRANSITION}

To investigate the unusual change in the pressure dependence of the graphitelike modes at approximately $1.7 \mathrm{GPa}$, we have carried out a series of molecular dynamic simulations. Because of the large size of the unit cell and the large configuration space, such calculations are very costly using either $a b$ initio or tight-binding total-energy calculations. We have used the universal force field (UFF) of Rappe et $a l^{33}$ via the CERIUS2 molecular modeling software to calculate the lattice constants of the nanotube bundle as a function of pressure. This model uses classical two-, three-, and four-body potentials to compute the interaction energy of atoms in a molecule or between atoms of different molecules (i.e., bonded and nonbonded interactions, respectively). Specifically, it contains terms for bond stretching, bond-angle bending, dihedral-angle torsion, and also electrostatic and van der Waals interactions. These last two interactions only apply to atoms not bonded to each other and not connected via a single common atom.

In Fig. 8 we show the equilibrium lattice constants of a $(10,10)$ nanotube bundle as functions of pressure. The atomic positions and lattice constants are relaxed to minimize the total energy at each pressure as computed by the UFF method. Due to the incompatibility of the structure of the $(10,10)$ tube with hexagonal symmetry, there is a small difference in the two in-plane lattice constants. Thus, the symmetry of the nanorope is monoclinic. As the pressure increases, this difference between the two lattice constants increases. As expected, the unit cell volume decreases continuously with pressure. However, at a pressure of $1.75 \mathrm{GPa}$, the lattice structure undergoes a sudden transition. This can be clearly seen in both the lattice constants and the unit cell volume. To examine the transition, we show the unit cell structures just before and just after the transition. See insert in Fig. 8. The nanotube morphology after the transition is deformed to a more oval shape while that before the transition has a more circular cross section. ${ }^{9}$ Thus, in the high pressure region a larger portion of the walls of adjacent tubes are approximately parallel, so the interaction between the tubes more closely resembles that of the interlayer interaction in graphite. It is then expected that the pressure dependence in the high-pressure region should resemble that found in bulk graphite more closely than the dependence seen in the low-pressure region. The fact that our experimental data show a clear kink around $1.7 \mathrm{GPa}$, lets us conclude that the kink is the first evidence that the nanotubes undergo a structural phase transition around this pressure.

Intuitively the structural transition can be understood as follows. At ambient pressure the lattice constants are such that the shortest distance between $\mathrm{C}$ atoms on neighboring tubes is close to the van der Waals distance (3.4 $\AA$ ). As the pressure increases the intertube distance shrinks while the tube geometry remains relatively intact. This is because the intertube van der Waals force is much smaller than the elastic force needed to distort the nanotube geometry. As the pressure increases the intertube distance hits the hard repulsive wall of the van der Waals interaction. Then it becomes energetically favorable to distort the nanotube geometry to reduce the intertube empty space to accommodate the pressure, rather than to simple continue to decrease the intertube distance. Since the lowest instability mode of nanotube distortion has a twofold symmetry ${ }^{21,8}$ the natural shape of a distorted nanotube is oval rather than the hexagonal shape one might expect. The oval nanotube shape leads to a different symmetry for nanoropes at high pressure. Thus, a structural transition occurs. The transition is a first-order one because there are discontinuities in both the lattice constants and the cohesive energy. The oval shape of the nanotubes also explains the fact that the breathing mode becomes unobservable at high pressure because it is no longer an eigenmode.

In conclusion, we have measured the frequency of the radial breathing modes and several of the graphitelike higher-frequency modes in the Raman spectrum of $(10,10)$ and $(17,0)$ carbon nanotubes as a function of applied pressure. We find that the response to pressure divides into two regions. For pressures below approximately $1.7 \mathrm{GPa}$, all of the measured modes shift to higher frequencies as the pressure is increased, and the slope of frequency vs pressure is the same for all modes. The measured slope is in good agreement with the value calculated using tight-binding methods. Above $1.7 \mathrm{GPa}$ the breathing mode becomes unobservable, and the slope of frequency vs pressure for the graphitelike 
modes decreases abruptly. For the two higher-frequency graphitelike modes, the value of the slope in this region is very similar to that found in corresponding modes in bulk graphite. The lowest-frequency mode of this group is essentially insensitive to pressure in this region. This difference in the response to pressure is explained by noting that the lowest-frequency mode involves only atomic motion parallel to the axis of the tube, which leads to a reduced sensitivity to the proximity of adjacent tubes. This is in contrast to the two upper modes, which involve circumferential atomic motion and thus a greater sensitivity to tube spacing.

We have analyzed the abrupt change in the slope of frequency vs pressure observed in these high-pressure Raman spectroscopy measurements by means of molecular dynamics simulations, which show a structural phase transition at 1.7 GPa. In this transition the lattice constants and the unit cell volume change abruptly as the nanotubes deform from a circular to an oval cross section.

\section{ACKNOWLEDGMENTS}

The authors thank J. Lorentzen, C. Bower, and O. Zhou for their help with this project. This work was supported by the Office of Naval research through a MURI program (Grant No. N0001-98-1-0597).
${ }^{1}$ S. Iijima, Nature (London) 354, 56 (1991).

${ }^{2}$ J. W. Mintmire, B. I. Dunlap, and C. T. White, Phys. Rev. Lett. 68, 631 (1992).

${ }^{3}$ N. Hamada, S. Sawada, and A. Oshiyama, Phys. Rev. Lett. 68, 1579 (1992).

${ }^{4}$ L. Wang, P. S. Davids, A. Saxena, and A. R. Bishop, Phys. Rev. B 46, 7175 (1992).

${ }^{5}$ R. Saito, M. Fujita, G. Dresselhaus, and M. S. Dresselhaus, Phys. Rev. B 46, 1804 (1992).

${ }^{6}$ D. H. Robertson, D. W. Brenner, and J. W. Mintmire, Phys. Rev. B 45, 12592 (1992).

${ }^{7}$ M. M. Treacy, T. W. Ebbesen, and J. M. Gibson, Nature (London) 381, 678 (1996).

${ }^{8}$ B. I. Yakobson, C. J. Brabec, and J. Bernholc, Phys. Rev. Lett. 76, 2511 (1996).

${ }^{9}$ S. A. Chesnokov, V. A. Nalimova, A. G. Rinzler, R. E. Smalley, and J. E. Fischer, Phys. Rev. Lett. 82, 343 (1999).

${ }^{10}$ Jian Ping Lu, Phys. Rev. Lett. 79, 1297 (1997).

${ }^{11}$ W. Li, H. Zhang, C. Wang, Y. Zhang, L. Xu, K. Zhu, and S. Xie, Appl. Phys. Lett. 70, 2684 (1997).

${ }^{12}$ U. D. Venkateswaran, A. M. Rao, E. Richter, M. Menon, A. Rinzler, R. E. Smalley, and P. C. Ecklund, Phys. Rev. B 59, 10928 (1999).

${ }^{13}$ A. V. Bazhenov, V. V. Kvedar, A. A. Maksimov, I. I. Tartakovskii, R. A. Oganyan, Yu A. Ossipyan, and A. I. Shalynin, J. Exp. Theor. Phys. 86, 1030 (1998).

${ }^{14}$ Wenzhi Li, Hao Zhang, Chaoying Wang, Yun Zhang, Liwen Xu, Ke Zhu, and Sishen Xie, Appl. Phys. Lett. 70, 2684 (1997).

${ }^{15}$ N. Chandrabhas, A. K. Sood, D. Sundararaman, S. Raju, V. S. Raghunathan, G. V. N. Rao, V. S. Sastry, T. S. Radhakrishnan, Y. Hariharan, A. Bharathi, and C. S. Sundar, Pramana J. Phys. 42, 375 (1994)

${ }^{16}$ H. Hiura, T. W. Ebbesen, and K. Tanigaki, Chem. Phys. Lett. 202, 509 (1993).

${ }^{17}$ M. Sugano, A. Kasuya, K. Tohji, Y. Saito, and Y. Nishina, Chem. Phys. Lett. 292, 575 (1998).
${ }^{18}$ A. Kasuya, M. Sugano, T. Maeda, Y. Saito, K. Tohji, H. Takahashi, Y. Sasaki, M. Fukushima, Y. Nishina, and C. Horie, Phys. Rev. B 57, 4999 (1998).

${ }^{19}$ M. A. Pimenta, A. Marucci, S. A. Empedocles, M. G. Baawendi, E. B. Hanlon, A. M. Rao, P. C. Ecklund, G. Dresselhaus, and M. S. Dresselhaus, Phys. Rev. B 58, R16 016 (1998).

${ }^{20}$ A. M. Rao, E. Richter, S. Bandow, B. Chase, P. C. Ecklund, K. A. Williams, S. Fang, K. R. Subbaswamy, M. Menon, A. Thess, R. E. Smalley, G. Dresselhaus, and M. S. Dresselhaus, Science 275, 187 (1997).

${ }^{21}$ Daniel Kahn and Jian Ping Lu, Phys. Rev. B 60, 6535 (1999).

${ }^{22}$ R. Saito, T. Takeya, T. Kimura, G. Dresselhaus, and M. S. Dresselhaus, Phys. Rev. B 57, 4145 (1998).

${ }^{23}$ Ernst Richter and K. Subbaswamy, Phys. Rev. Lett. 79, 2738 (1997).

${ }^{24}$ Jin Yu, Rajiv K. Kalia, and Priya Vashishta, J. Chem. Phys. 103, 6697 (1995).

${ }^{25}$ J. Yu, K. Kalia, and P. Vashishta, J. Chem. Phys. 103, 6697 (1995).

${ }^{26}$ A. Kasuya, Y. Sasaki, Y. Saito, K. Tohji, and Y. Nishina, Phys. Rev. Lett. 78, 4434 (1997).

${ }^{27}$ D. Sanchez-Portal, E. Artacho, J. M. Soler, A. Rubio, and P. Ordejon, Phys. Rev. B 59, 12678 (1999).

${ }^{28}$ C. Bower, S. Suzuki, K. Tanigaki, and O. Zhou, Appl. Phys. A: Mater. Sci. Process. 67, 47 (1998).

${ }^{29}$ G. J. Piermarini, S. Block, J. D. Barnett, and R. A. Forman, J. Appl. Phys. 46, 2774 (1975).

${ }^{30}$ J. Kurti, G. Kresse, and H. Kuzmany, Phys. Rev. B 58, R8869 (1998).

${ }^{31}$ M. S. Dresselhaus, G. F. Dresselhaus, and P. C. Ecklund, Science of Fullerenes and Carbon Nanotubes (Academic, New York, 1996), p. 17.

${ }^{32}$ N. Wada, Phys. Rev. B 24, 1065 (1981).

${ }^{33}$ A. K. Rappe, C. J. Casewit, K. S. Colwell, W. A. Goddard III, and W. M. Skiff, J. Am. Chem. Soc. 114, 10024 (1992). 